\title{
Analysis of transmit-receive diversity in Rayleigh fading
}

\begin{abstract}
We analyze the error performance of a wireless communication system employing transmitreceive diversity in Rayleigh fading. By focusing on the complex Gaussian statistics of the independent and identically distributed entries of the channel matrix, we derive a formula for the characteristic function (c.f.) of the maximum output signal-to-noise ratio. We use this c.f. to obtain a closed-form expression of the symbol error probability (SEP) for coherent binary keying. The method is easily extended to obtain the SEP for the coherent reception of M-ary modulation schemes.
\end{abstract}

Keyword: Coherent binary keying; Coherent M-ary modulation; Rayleigh fading; Symbol error probability (SEP); Transmit-receive diversity 